\title{
The Evolution of Knowledge: Rethinking Science in the Anthropocene ${ }^{1}$
}

\author{
Jürgen Renn \\ Max Planck Institute for the History of Science \\ renn@mpiwg-berlin.mpg.de
}

\begin{abstract}
The paper argues that humanity has entered a new stage of evolution: epistemic evolution. Just as cultural evolution emerged against the background of biological evolution, epistemic evolution began as an aspect of cultural evolution and now dominates the global fate of humanity. It is characterized by the increasing dependence of global society on the achievements and further extension of science and technology in order to ensure its sustainability in the age of the Anthropocene. The historical development of knowledge is reviewed from an evolutionary perspective that introduces key concepts of an historical epistemology.
\end{abstract}

Keywords: epistemic evolution, cultural evolution, Anthropocene, global society

\footnotetext{
${ }^{1}$ This paper is based on excerpts from: Jürgen Renn, The Evolution of Knowledge: Toward a Historical Theory of Human Thinking (Princeton: Princeton University Press, forthcoming in 2019), and published with kind permission of Princeton University Press. It was presented in Lisbon as the $1^{s t}$ CIUHCT (Centro Interuniversitário de História das Ciências e da Tecnologia) Distinguished Lecture, "Knowledge in the Anthropocene," at the Faculdade de Ciências da Universidade de Lisboa, on November 8, 2017.
}

HoST - Journal of History of Science and Technology 12, pp. 1-22 


\section{Introduction}

Humans have changed the planet. Actually, humans have drastically changed the planet, with dramatic consequences. There remains virtually no untouched nature. ${ }^{2}$ A large part of the Earth's surface not covered by ice has been transformed by humans. The polar ice is melting, the water level of the oceans is rising and coastal and marine habitats are undergoing massive changes. More than half of Earth's fresh water is being exploited by humans. Oceans are being acidified and contaminated by aquacultures. Agricultural soil is being degraded. Beneath the surface, the earth is being altered by mines and drilling. The construction of thousands of dams and extensive deforestations massively affect water circulation and erosion rates and thus the evolution and geographical spread of numerous species. The loss of biodiversity is greater by orders of magnitudes than it would be without human intervention. On average, at least every third nitrogen atom in the biosphere has been once processed by the fertilizer industry. Most of the biomass of all living mammals is constituted by humans and domesticated animals.

Through energy-intensive chemical processes, humans have created functional materials (which are rare under natural conditions), and brought them into wide circulation. Among these are elemental aluminum, lead, cadmium, and mercury, fly ash residues from the high-temperature combustion of coal and oil, and also concrete, plastic, and other man-made materials, many of them displaying properties alien to the natural world. Plutonium from atmospheric nuclear testing will persist in the sedimentary record for the next several 100,000 years while it decays into uranium and then into lead. We are measuring the highest atmospheric concentrations of the greenhouse gases carbon dioxide and methane in at least 800,000 years. Even if the use of fossil energy resources were to stop immediately, it would take thousands of

\footnotetext{
${ }^{2}$ For the following, see Jeremy Davies, The Birth of the Anthropocene (Oakland, CA: University of California Press, 2016); Jürgen Renn and Bernd Scherer, Das Anthropozän: Zum Stand der Dinge (Berlin: Matthes \& Seitz, 2015); W. Steffen, A. Sanderson, P.D. Tyson, J. Jäger, P.A. Matson, B. Moore III, F. Oldfield, et al., Global Change and the Earth System: A Planet Under Pressure (Heidelberg, New York: Springer, 2004); Helmuth Trischler, "The Anthropocene," NTM Zeitschrift für Geschichte der Wissenschaften, Technik und Medizin 24, no. 3 (2016): 309-335; Paul Voosen, "Anthropocene Pinned to Postwar Period," Science 353, no. 6302 (2016): 852-853.
}

HoST - Journal of History of Science and Technology 12, pp. 1-22

DOI 10.2478/host-2018-0001 
years before that concentration sank to pre-industrial levels.

Some changes have occurred at a much brisker pace than natural processes. The present concentration of carbon dioxide has been reached at a rate at least ten, and possibly one hundred times faster than increases at any time during the previous 420,000 years. Simultaneously, new diseases spread via carriers with rapid life cycles that allow them to adapt quickly to the new conditions. How quickly will human societies be able to adapt to the same? The ongoing changes will affect, in any case, different parts of the globe in different ways and the global nature of these changes will not always be easy to recognize for those who suffer from them. With floods increasingly menacing lowlying cities near large bodies of water, new forms of gentrification emerge, raising the prices of dry and secure locations and displacing the poor. Previously fertile farmlands dry out because of drought, inciting allocation battles and migrations to richer nations. Developed countries may actually appear to benefit from climate change, while developing countries suffer-but ultimately everyone will lose. There will be no escape, not even for the rich.

In short, the planet is changing with irreversible consequences. There is no hope that we might ever be able to return to some "natural state" of affairs. Humanity has affected the energy balance at the Earth's surface, resulting in the transition of our planet into a new stage. Humanity does not act against the backdrop of an unchangeable nature; it is deeply woven into its very fabric, shaping both its imminent and distant future. The fundamental revision of our understanding of the state of this planet may only be compared with the upheaval of our physical conceptions of space and time in the wake of Einstein's theories of relativity. In classical physics, space and time seemed to be the rigid stage on which world events were taking place. According to Einstein's theory, in contrast, this stage is no immutable framework is itself part of the drama; there is no absolute distinction between the actors and the scenery. Space and time do not remain in the background of physical processes but rather take part in them. The new reality of the planet confronts us with a similarly radical need for rethinking our situation: we are not living in a stable environment that simply serves as a stage and resource for our actions, but we are all actors in a comprehensive drama in which humans and the nonhuman world equally take part.

HoST - Journal of History of Science and Technology 12, pp. 1-22

DOI 10.2478/host-2018-0001 


\section{Introducing the Anthropocene}

In the year 2000, Nobel Prize winner and discoverer of the hole in the Ozone layer Paul J. Crutzen felt uneasy with the official account of the state of the planet, according to which we are presently living in the "Holocene" epoch. Geologists have a sophisticated system to organize the enormous time span of the Earth's history into intervals. Holocene means "entirely recent" and is the second epoch of the so-called Quaternary Period. It may sound strange, the Quaternary is actually an Ice Age that began 2.6 million years ago; more precisely, the Quaternary is characterized by a backand-forth of polar ice. The Holocene is an interglacial period in which the ice retreats. It began at the end of an anomalous cooling episode that interrupted postglacial warming, dated by radiocarbon to between ca. 12,900 and 11,500 years BP (before the present). Its temperatures have been strikingly stable for more than 11,000 years. Crutzen was participating in a conference on Earth system science outside Mexico City when he was struck by a sudden dislike for the Holocene description, which seemed to utterly belittle the human impact on the Earth system. He told the delegates to stop using the term Holocene and, while speaking, searched for a better term: "We are not in the Holocene anymore, we are in the, the, the Anthropocene!"3

As it turned out, the term had been used by the limnologist Eugene F. Stoermer since the 1980s. ${ }^{4}$ Similar terms have been introduced independently by several scientists. Its roots go back even to the eighteenth century, when the French naturalist Buffon remarked that "the entire face of the Earth bears the impact of human power." Its current centrality for a broad discourse on planetary changes and humanity's role in them are, however, connected with that crucial moment in the year 2000. Crutzen himself and scholars of many disciplines have since controversially discussed the Anthropocene and its meaning in understanding humanity's predicament, but also its viability as a geologic time period. The term is currently being examined by the Anthropocene

\footnotetext{
${ }^{3}$ Davies, The Birth of the Anthropocene; Christian Schwägerl, The Anthropocene: The Human Era and How It Shapes Our Planet (Santa Fe, NM: Synergetic Press, 2014).

4 Paul J. Crutzen and Eugene F. Stoermer, "The 'Anthropocene'," Global Change Newsletter no 41 (2000): 17-18.

${ }^{5}$ Quoted after Trischler, “The Anthropocene," an excellent survey of the current discussion.
}

HoST - Journal of History of Science and Technology 12, pp. 1-22

DOI 10.2478/host-2018-0001 
Working Group, ${ }^{6}$ which will submit a formal proposal to the Subcommission on Quaternary Stratigraphy, which in turn reports to the International Commission on Stratigraphy, itself responsible to the International Union of Geological Sciences where it will be ratified by its Executive Committee. ${ }^{7}$ Whatever the final decision of the geological experts may be, the concept of the Anthropocene has opened our eyes towards a fundamentally altered global environment and the fact that humanity has meanwhile changed the planet to a degree comparable to geologic forces.

Given the massive impact of human interventions in the planetary environment, the traditional line between nature and culture has become problematic. We are living in an "anthropological nature" (Karl Marx) resulting from our own interventions. ${ }^{8}$ Furthermore, the timeline of human history has become intrinsically meshed with the geologic timeline. Our economic metabolism feeds on fossil energy, consuming within a time span of hundreds of years resources that have been created over hundreds of millions of years. Just as geologic time is turned into historical time, our impact as a geologic force turns human history into a significant part of geologic history.

\section{Who is destroying our planet?}

The transformative power of humanity is evidently based on knowledge, accumulated and implemented over generations. What has also accumulated, however, are the unintended consequences of human activity. Nobody seriously intended to destroy the planet! Specifically, science and technology have contributed much to bring us into this situation. Global capitalism, industrialization, traffic, and population growth would not

\footnotetext{
${ }^{6}$ quaternary.stratigraphy.org/working-groups/anthropocene/ (accessed September, 5, 2018).

7 Voosen, "The Anthropocene Pinned."

${ }^{8}$ Karl Marx, Economic and Philosophic Manuscripts of 1844, 143 (London: Lawrence \& Wishart, 1970). For a discussion of the challenges for historical scholarship, see also Dipesh Chakrabarty, "The Climate of History: Four Theses," Critical Inquiry 35, no 2 (2009), 197-222.
} 
be possible without the advances of science and technology. ${ }^{9}$ They have catapulted us from the age of horse-drawn plows and carriages to the age of industrialized agriculture and self-driving cars, and they have blessed us with the unequally distributed benefits of modern medicine. But along with their progress, science and technology have generated uncontrolled growth, the ruthless exploitation of natural resources, and a rapid increase of the greenhouse emissions that are changing the global climate. Humans are now able to send missions into interplanetary space but they have not yet found a way to protect billions from poverty or starvation, to contain wars, or to cope with all the other challenges of the Anthropocene for humanity as a whole. True, these are political and economic questions, not just questions of knowledge, but they are also questions of knowledge and of science.

Rethinking science in the Anthropocene means to ask a number of fundamental questions about science and knowledge, as well as about their historical evolution. In this paper, I proceed in five steps: I have already begun to discuss the double character of knowledge crucial for our entry into the Anthropocene, the empowerment it provides, and the unintended consequences it leads to, which I shall briefly recapitulate below. This is the first step. In a second step, I shall discuss the historical nature of thinking and will ask how knowledge structures change. In the third part, dedicated to the "economy of knowledge," I shall investigate how knowledge structures affect society and how society affects knowledge structures. In the fourth part, I shall analyze diffusion and globalization processes of knowledge, asking how knowledge spreads. Finally, in the fifth part, I shall come back to the theme of the Anthropocene, discussing its emergence against the background of evolutionary considerations and asking the question on what knowledge our future depends.

Let me once more recapitulate the role of knowledge in and for the Anthropocene: Humans have massively intervened in various Earth system cycles, such as the carbon cycle, causing climate change, as well as the water, nitrogen, phosphorus and sulphur

\footnotetext{
9 See Jürgen Renn, Manfred D. Laubichler, and Helge Wendt, "Energietransformationen Zwischen Kaffee und Koevolution," in Willkommen Im Anthropozän! Unsere Verantwortung für die Zukunft der Erde, Katalog zur Sonderausstellung am Deutschen Museum, ed. Nina Möllers, Christian Schwägerl, and Helmuth Trischler, 81-84 (Munich: Deutsches Museum, 2014).
} 
cycles, all of which are fundamental to life on Earth. Humanity has affected the energy balance at the Earth's surface, resulting in the transition of our planet into a new stage, the Anthropocene, propelled and empowered by the knowledge embodied in our technologies and our material culture. The question of whether and when the Anthropocene began is still debated. What is clear is that this knowledge has accumulated over generations and ever more quickly since the Scientific Revolution, the Industrial Revolution, and the so-called Great Acceleration of the 1950s. ${ }^{10}$

\section{The Great Acceleration}

The human species has mushroomed all over the planet. For all of humanity's interventions in planetary cycles, it is still part of the biosphere, with no power to transcend it. We are not outside observers! What distinguishes humanity from the rest of the biosphere is cultural evolution, a unique layer of metabolism and learning on top of biological evolution. With their rapidly evolving culture, humans have introduced an "ergosphere" (a sphere of work, as well as of technological and energetic transformations) as a new global component of the Earth system, in addition to the lithosphere, the hydrosphere, the atmosphere, and the biosphere, thus changing the overall dynamics of the system.

Unwisely, we have left the astonishingly stable state of the Holocene, which has shaped our culture and our ways of thinking. The Anthropocene may be a contrary state of the Earth system, but it is not necessarily the demise of humanity - we simply do not yet know what it holds in store for us. By leaving our "Holocenic bubble," we are not withdrawing from a static system but rather intervening within a system that is itself highly dynamic. We are performing a global experiment on a system that is already changing itself; our interventions therefore introduce second-order changes. As a consequence, we make ourselves ever more dependent on our understanding of this complex dynamic system and our interactions with it. This understanding is itself not

${ }^{10}$ See Will Steffen, Wendy Broadgate, Lisa Deutsch, et al., "The Trajectory of the Anthropocene: The Great Acceleration," The Anthropocene Review 2, no 1 (2015): 81-98.

HoST - Journal of History of Science and Technology 12, pp. 1-22

DOI 10.2478/host-2018-0001 
static but subject to a dynamical evolution. Understanding the dynamics of knowledge is therefore crucial for our survival in the Anthropocene. Both knowledge and changes in the environment accumulate across generations in long-term processes, and not necessarily in such a way that human survival guaranteed.

\section{The Architecture of Knowledge}

But what is knowledge? This question brings me to part two. Individual knowledge is based on encoding experiences, enabling individuals to solve problems as part of their adaptive behavior. It is rooted in the ability to anticipate actions and their results, and can be corrected in response to consequences since we can think or "reflect" on our experiences. Due to the dependence of knowledge on prior experience, its predictive power is in principle limited. On the other hand, knowledge can be mentally stored in the form of cognitive structures and repurposed for new goals. Although we are describing cognitive structures of human thinking on a purely phenomenological level, we can observe a number of remarkable aspects.

One of these aspects is that the structure and content of knowledge are not as independent from each other as a long tradition separating logic from meaning has held. Any concept (e.g. the concept of a tree) may specifically refer to a concrete experience and, at the same time, act as a general cognitive structure to which this experience can be assimilated, connecting it to a web of other experiences. If one thinks about it, every concept is a little theory because it is semantically connected to other concepts.

Cognitive structures are active in the sense that they change with every implementation. If I see a tree I have never seen before, I may change my concept of a tree and thus the entire network of semantic relations in which this concept is embedded. This is a fundamental feature of cognitive development that also accounts for how scientific knowledge may change due to a novel experience, in particular, when this experience is difficult to understand, taking a particular form that we designate as "challenging

HoST - Journal of History of Science and Technology 12, pp. 1-22

DOI 10.2478/host-2018-0001 
objects." ${ }^{11}$ For example, when one expects that all physical interactions require direct contact between bodies, the invisible forces moving the needle of a compass may appear miraculous, as they did for the young Einstein, who remembered this encounter all his life as a challenging experience.

Cognitive structures allow for thinking processes even under circumstances of incomplete information, which is our typical predicament. Lacking information in parts of the structure may be supplemented by information gleaned from prior experience in the form of "default assumptions," such as when I see only the upper part of a tree and conclude that it must have roots because trees normally do. If it turns out, however, that the roots have been cut, I do not have to abandon my conviction that I am dealing with a tree. Surrealist art plays with our default expectations, baffling us, for instance, by painting a sailing ship with butterflies where we expect sails according to our "mental model" of a sailing ship. ${ }^{12}$

The availability of default settings and the flexibility in replacing them is an important feature also of scientific heuristics. It helps us to understand why scientific theories do not have to be abandoned in the face of contrary evidence: it is enough to simply replace some of the default settings while maintaining the essential argumentative structure. Incomplete scientific information is typically complemented by background knowledge consisting of default assumptions rooted in other prior knowledge. When Galileo saw a blurry picture of the moon in his self-made telescope, he could identify its irregularities as craters and mountains due to his prior experience with landscape images and his expectation of seeing another Earth-like body.

The components of a cognitive structure may be given by other cognitive structures, as when the concept of a house is explicated in terms of its basement, floor, roof, walls,

\footnotetext{
${ }^{11}$ See Jürgen Renn, Peter Damerow, and Simone Rieger, "Hunting the White Elephant: When and How did Galileo Discover the Law of Fall? (with an Appendix by Domenico Giulini)," in Galileo in Context, ed. J. Renn, 29-149 (Cambridge: Cambridge University Press, 2001).

${ }^{12}$ See Jürgen Renn, Peter Damerow, Matthias Schemmel, Christoph Lehner, and Matteo Valleriani, "Mental Models as Cognitive Instruments in the Transformation of Knowledge," in Emergence and Expansion of Pre-Classical Mechanics, ed. R. Feldhay, J. Renn, M. Schemmel, and M. Valleriani (Dordrecht: Springer, 2018).
} 
windows, doors, and so forth, with each of these concepts in turn being expandable in terms of its components, such as a door's knob, hinges, lock, wood panel, paint, and so on. Cognitive structures are thus somewhat like Russian dolls, with a nested sequence of other cognitive structures inside. But these hierarchies are not fixed so that one could also start from "inside the box," as it were, expanding it in another direction. For instance, when starting from the concept of a wood panel one might find inside its "Russian doll sequence" the example of a door made out of wooden panels, among many other things. In other terms, we can speak of semantic networks that may be arranged in different hierarchies. Altering such hierarchies is in fact another salient feature of conceptual change in science. An originally marginal concept within a given system may eventually find itself at the center of a new conceptual system. Inertial motion, for example, had long been noted when a projectile leaves the hurling motion of a slingshot along a straight line, but it became an explicitly defined anchor point of a conceptual system only with classical mechanics.

We refer to these complex interdependencies as the "architecture of knowledge." Two further general features are important: There is a certain layered structure to this architecture due to the fact that some experiences, such as those of our moving around in the material world, are rather basic and persistent: lifting heavy objects requires effort, rigid walls are hard to penetrate, and so on. These underlying layers of "intuitive knowledge" often provide default settings for expectations when entering new terrain or when dealing with less concrete matters, where they often reappear as metaphors. The other general observation is that people strive for a mental representation of their world that seems rather complete. To some extent, of course, all of us are aware of our blind spots, but we nevertheless tend to avoid an "epistemic vacuum," and thus complement our partial knowledge of the world with default assumptions, striving for a more-or less-holistic view of the world around us.

Knowledge has not only mental, but also social and material dimensions. It can be stored, shared, and passed on from individual to individual and across generations with the help of "external representations," such as writing or symbol systems, which are part of the material culture of a society. Material culture not only determines horizons of possible actions, and forms of social organization, but also a horizon of 
thinking. The emergence of the concept of energy, for instance, only became possible once actual transformations of motive power (e.g. the replacement of human force by wind or waterpower and later by the steam engine) emerged historically as material practices.

External representations such as writing or symbol systems may serve as "paper tools"13 to support "reflection" in the sense of thinking on thinking: reflecting on actions with such external representations (i.e., on symbols representing objects to be counted) may engender "higher-order forms of knowledge," (e.g. an abstract concept of number) a process we refer to as "reflective abstraction." These higher-order forms of knowledge may seem to be decoupled from the primary objects of experience, but they actually remain related to them by historically specific knowledge transformations. The resulting abstract concepts, such as the concepts of number, energy, chemical element, or gene, serve to "integrate" numerous experiences within a conceptual system (e.g. the conceptual system of thermodynamics or of modern biology). All of their concepts remain, of course, liable to further change. ${ }^{14}$

Knowledge is typically part of "systems of knowledge." One example is the curriculum of the medieval university, with its faculties of theology, medicine, and jurisprudence, prepared for by prior study of the seven liberal arts. Another example is the modern system of scientific disciplines. Systems of knowledge in our sense do not have to be conceptual systems integrated by abstract concepts, as in the examples above; in fact, they do not have to be very systematic at all. The knowledge needed to build a house or to tend a garden may also be conceived as a system of knowledge, composed of many different elements. These elements are not held together by a strict organizational principle, but rather constitute a heterogeneous "package of knowledge." The relations

\footnotetext{
13 See Ursula Klein, "Paper Tools in Experimental Cultures," Stud. Hist. Phil. Sci. 32, no. 2 (2001): 265-302, and Experiments, Models, Paper Tools: Cultures of Organic Chemistry in the Nineteenth Century (Stanford: Stanford University Press, 2003).

14 See Peter Damerow and Wolfgang Lefevre, "Wissenssysteme im geschichtlichen Wandel," in Enzyklopädie der Psychologie: Themenbereich C: Theorie und Forschung. Serie II: Kognition: Vol. 6: Wissen, ed. F. Klix and H. Spada, 77-113 (Göttingen: Hogrefe Verlag, 1998).
}

HoST - Journal of History of Science and Technology 12, pp. 1-22

DOI 10.2478/host-2018-0001 
among the components of a system of knowledge may be semantic, as in a scientific theory, they may be institutional, as in a curriculum, or they may be practical, as in the example of a building project.

Scientific knowledge is a special form of knowledge, involving not just theories but cultural practices. It first emerged in complex societies that created social spaces for exploring knowledge independent from immediate practical purposes. No one could have anticipated that human societies would eventually become dependent on such knowledge. Scientific knowledge is accumulated and transmitted by "epistemic communities" dealing —often within dedicated institutions—with the preservation, improvement, and generation of knowledge. We may speak of "science," for instance, whenever the potential inherent in the material or symbolic culture of a society is being explored for the primary sake of knowledge generation. Scientific knowledge is rooted in other forms of knowledge, such as practical knowledge, but is encoded in special ways, such as writing.

Scientific practice comprises forms of initiation, education, exploration, discourse, and transmission that are subject to historical change. Historically variable argumentative standards, control structures, and practices for the validation of knowledge shape its accumulation and corrigibility, extending the learning and self-correcting aspects of individual knowledge to a societal institution. The form which scientific knowledge takes also depends on the role a society assigns to knowledge, its "image of knowledge" (Yehuda Elkana), and the criteria applied for its validation.

Scientific systems of knowledge are subject to persistent change in long-term processes of accumulation, loss, and transformation, which may occasionally give rise to farreaching reorganizations of both the architecture of knowledge and the relevant epistemic communities. Some of these transformation processes are traditionally called "scientific revolutions." However, they do not result from "paradigm shifts" (Thomas Kuhn) but rather from more transformative processes, such as the exploration of an existing system of knowledge to its limits, which may give rise to a "matrix" for the emergence of a new system. Such reorganizations of knowledge systems open up new perspectives as well as possibilities for disruptive societal change that cannot 
be predicted by any "linear model" or scientific and technological progress. For this reason alone, focusing all resources on applied research dealing with evident global challenges would make little sense. Grand challenges do not automatically generate grand solutions, and changes of perspectives and disruptive innovations can hardly be anticipated.

\section{The Relation Between Knowledge and Society}

The third part of this paper deals more systematically with the relation between knowledge and society. We describe and analyze the relation between society and knowledge as two aspects of a common dynamics shaping human existence. A key mechanism of their interdependence is an "entrapment of mutual limitation." The mental and cognitive development of individuals is limited by the experiential horizon a society offers to them, while societal regulations are constrained by a limited range of individual mental capabilities and perspectives. This interdependence also shapes the capability of individuals and societies to maintain complex forms of cognitive and social organization, withstanding the ever-present temptation of self-destruction. Societal changes necessarily involve a co-evolution of societal and mental structures.

Just as the technology of material practices allows for the formation of scientific abstractions, like the concept of energy, societal practices like administrations or markets may give rise to "cultural abstractions," such as the concepts of time and economic value (Norbert Elias, Karl Marx). External representations of these cultural abstractions, such as clocks or money, may in turn become a starting point for new social regulations and developments, such as the rise of capitalism. With the increasing societal role of information technologies, data have become a key role cultural abstraction steering societal and economic processes. Cultural abstractions shape our thinking and our social existence. They are not easy to change because they are the result of the evolution of complex systems that are highly path dependent. Yet, as the history of science shows, 
they can be changed after all. ${ }^{15}$

While all abstractions tend to be opaque with regard to the historically specific experiences from which they emerge, they may be directly challenged by new experiences. In the history of science, such challenges have triggered major upheavals of fundamental concepts such as space, time, and matter. In societal development, the experiences and challenges of the Anthropocene may also warrant new cultural abstractions as regulatory mechanisms for coping with problems of planetary boundaries and limited resources. Concepts such as "carbon footprint," for instance, may help to regulate the carbon dioxide balance of the Earth system.

Every society has a "knowledge economy." 16 It designates the ensemble of its social institutions and processes, producing and reproducing the knowledge at its disposal, and, in particular, the knowledge on which its reproduction as a society relies. The external representations available to a society, especially its "knowledge representation technologies," be they papyrus, parchment, paper, or the Web- shape its knowledge economy. The action potential of a society (i.e., its reaction to external challenges) depends on its knowledge economy. While knowledge enables individuals to plan their actions and consider the results, a society cannot "think" but can only anticipate the consequences of its actions within its economy of knowledge. The ability of the global human society to cope with the challenges of the Anthropocene will therefore critically depend on the development of its knowledge economy.

The production of scientific knowledge is regulated by a knowledge economy separate from the immediate needs of societal reproduction, and yet has become an existential condition of global society. The internal regulation of this separate knowledge economy as well as the interfaces shaping the societal implementation of scientific knowledge have become critical factors in humanity's ability to cope with the Anthropocene.

\footnotetext{
15 See Jürgen Renn, “The Globalization of Knowledge in History and its Normative Challenges," Rechtsgeschichte/Legal History 22 (2014): 52-60.

16 See Jürgen Renn and Malcolm D. Hyman, "The Globalization of Knowledge in History: An Introduction," in The Globalization of Knowledge in History, ed. J. Renn, 15-44, 23 (Berlin: Edition Open Access, 2012): http://www.edition-open-access.de/studies/1/5/index.html\#44 (accessed September, 5, 2018).
}

HoST - Journal of History of Science and Technology 12, pp. 1-22

DOI 10.2478/host-2018-0001 
Given the unpredictability and inevitable serendipity of innovations, it would be shortsighted to bend the practice of science primarily toward the challenges at hand. It would be equally risky, on the other hand, to maintain the self-inflicted fragmentation characteristic of the current knowledge economy, as driven by the competition for real and "symbolic" capital.

The fragmentation of scientific knowledge due to disciplinary specialization and compartmentalization, traditional publication venues, commercialization, and so forth, may hinder opportunities for knowledge integration and implementation, blocking urgently needed innovations. The Web, on the other hand, offers a knowledge representation technology with the potential to optimize the current knowledge economy toward a global co-production of knowledge and new forms of organizing, integrating, locally adapting, and implementing scientific knowledge.

A future Web of Knowledge or an "Epistemic Web" would help to balance asymmetries in the ownership and control of knowledge and allow users to become "prosumers," for instance, by replacing browsers with interfaces optimized for interacting with global human knowledge as represented on the Web. ${ }^{17}$ Not only content but also the network of links would have to become an openly accessible good. A new representation technology would have to co-evolve with a new economy of scientific knowledge, and it would have to include a reward system focusing on the actual knowledge generated. On an Epistemic Web, this knowledge would be directly accessible, traceable, and reviewable. Such a level of transparency would enhance trust in the endeavor of science and would open up new opportunities for cooperation with other societal players. Globally sharing and co-producing knowledge may also help to overcome the entrapment by mutual limitation mentioned above through the opening up of new experiential spaces.

\footnotetext{
${ }^{17}$ Malcolm D. Hyman and Jürgen Renn, "Toward an Epistemic Web," in The Globalization of Knowledge in History, ed. J. Renn, 821-838 (Berlin: Edition Open Access, 2012): http://edition-open-access.de/ media/studies/1/36/Studies1ch32.pdf (accessed September, 5, 2018).
}

HoST - Journal of History of Science and Technology 12, pp. 1-22 


\section{Circulation of Knowledge and its Mechanisms}

The fourth part of this paper deals with the spread of knowledge and the mechanisms of knowledge transfer. Globalization in the sense of the potentially global spread of means of social cohesion (ranging from economy to culture, knowledge, and science) is a layered process. In the course of history, globalization processes have created "sediments" such as the global spread of basic technologies (e.g. food production or writing) that change the conditions for subsequent globalizations. I argue that globalization processes do not necessarily lead to a homogenization of the world because of the crucial role of knowledge, in particular its role in shaping the identity of the actors in such processes. Knowledge transfer always involves an active appropriation on the "receiving" end, associated with the generation of new local knowledge.

The transfer of knowledge between societies is shaped by their respective knowledge economies, with the consequence that knowledge transfer generally amounts to a knowledge transformation, perhaps through its modification to the needs of the receiving knowledge economy or a hybridization of existing and new knowledge. One example is the transfer of European scientific knowledge to China in the early modern period and its assimilation into the Chinese economy of knowledge. ${ }^{18}$ Due to the embedding of knowledge within systems of knowledge, knowledge transfer may develop self-reinforcing dynamics as elements of the transferred knowledge point to parts still missing. This may be observed, for instance, in the various translation movements shaping the global history of knowledge.

The re-contextualization associated with knowledge transfer may lead to a "cultural refraction" in the sense of an explication of structures of knowledge implicit in the source knowledge economy. In the context of the disputation culture of ancient Greece, for instance, structures of practical knowledge of Babylonian and Egyptian origin were encoded in writing and made the object of explicit reflections. Cultural refraction may

\footnotetext{
${ }^{18}$ See Matthias Schemmel, "The Transmission of Scientific Knowledge from Europe to China in the Early Modern Period," in The Globalization of Knowledge in History, ed. J. Renn, 269-293 (Berlin: Edition Open Access, 2012): http://edition-open-access.de/media/studies/1/15/Studies1ch11.pdf (accessed September, 5, 2018).
}

HoST - Journal of History of Science and Technology 12, pp. 1-22

DOI 10.2478/host-2018-0001 
thus foster the emergence of higher-order knowledge with greater independence from local contexts and conditions. Modern science is the result of a long-term global history of knowledge, due to the formation of sediments of globalization processes as well as the cultural refractions just described. The so-called "Western" science is actually global science in two senses: First, it is a product of global history. Second, global history now depends on it.

The "globalization of knowledge" in history is driven by an intertwinement of intrinsic and extrinsic dynamics. ${ }^{19}$ The phrase extrinsic dynamics implies the spread of knowledge as a "fellow traveler" among other transfer processes, such as commerce, conquest, or missionary activity. Intrinsic dynamics arise due to the development of knowledge systems with an ever-broader experiential basis, such as those of modern science, empowering societies to extend their dominance due to technological, economic, and military superiority. This intertwinement accounts for the path dependency of the globalization of knowledge, in which accidental circumstances become ineluctable conditions of its further development. Even the nature of the most abstract and seemingly universal knowledge available to us is, in other words, shaped by a contingent history of globalization.

The spread of knowledge may be described in terms of "epistemic networks" that involve social, material, and semantic layers. ${ }^{20}$ These networks make it possible to account for self-organizing dynamics of the co-evolution of epistemic communities and systems of knowledge. The spread of knowledge over social networks (e.g., about a new experimental method) may lead to the proliferation of that knowledge and its enrichment within an initially loosely connected epistemic community. Novelties often come from outside an established system of knowledge. The community may in turn react to the enriched knowledge that is manifested by the network of corresponding external representations (e.g., a set of scientific papers), integrating the accumulated knowledge into a more strongly organized system of knowledge (e.g., a new scientific discipline) and reorganizing itself around it. It is through an iteration of this cycle

${ }^{19}$ Renn and Hyman, "The Globalization of Knowledge in History: An Introduction," 29: http://www. edition-open-access.de/studies/1/5/index.html\#64_(accessed September, 5, 2018).

${ }^{20}$ Ibid, 28. 
of proliferation and integration that distinctive systems of knowledge and epistemic communities organized around them may emerge from weakly connected semantic and social networks.

This also describes the socio-epistemic dynamics underlying some of the so-called scientific revolutions characterized not only by intellectual upheavals but also by a reorganization of scientific communities. This co-evolution is not necessarily a fast process, however, as may be illustrated by the emergence of a scientific community focused on Einstein's general theory of relativity that took half a century. The effectiveness of this dynamic evidently depends on the systems of knowledge involved, as well as on the specific properties of the external representations and social networks. These considerations are of relevance when considering the possibility of refocusing the attention of scientific communities as well as of civil society on the current challenges of the Anthropocene.

\section{Back to the Beginning: Evolution of Knowledge and the Anthropocene}

The fifth part of this paper returns to the relation between the Anthropocene and the evolution of knowledge. The entry into a new state of the Earth system is not due to a single cause and cannot be tied to a particular event in human history. It can best be described in terms of a cascade of evolutionary processes, as a transition from cultural to epistemic evolution. In cultural evolution, human societies have entered relations of production dependent on their material culture. In epistemic evolution, human societies' interactions with the Earth system are now dependent on science-based technologies, such as the use of fossil fuels, nuclear power, artificial fertilizers, and genetic engineering. Without the empowerment of the means of production through scientific knowledge, humanity would not have entered the "Great Acceleration." Once the knowledge economy of science entered a positive feedback loop with the capitalist economy, a runaway effect transformed cultural evolution into a process ever more dependent on science-based technologies. What stone tools, hunting, gathering, and later food production, clothing, and the building of shelters were for the Holocene,

HoST - Journal of History of Science and Technology 12, pp. 1-22

DOI 10.2478/host-2018-0001 
science and technology are now for the Anthropocene: essential conditions of human life as we know it.

The transition from cultural to epistemic evolution suggests the development of knowledge as an important aspect of cultural evolution. In the transition from biological to cultural evolution, the role of "niche construction" (e.g. beavers constructing their dams) has been transformed from an aspect of biological evolution into an essential feature of cultural evolution, as the role of material culture and tool use for the very emergence of modern humans illustrates. ${ }^{21}$ In the transition from cultural to epistemic evolution, the role of scientific knowledge has been similarly turned from an aspect into a characteristic feature of novel evolutionary dynamics. ${ }^{22}$

But what role does knowledge play in cultural evolution? The direction in which science and technology develop is not fixed and is certainly not governed by a rigid logic of progress. Their development is rather subject to an interplay between cognitive and contextual factors that includes other forms of knowledge as well. The experiment we are performing with our planet generates both massive changes of the environment and new knowledge. Therefore, we will need to better understand how this co-evolution works if we want to be able judge our predicament in the Anthropocene and adjust our behaviors, including our knowledge economies, accordingly.

From an evolutionary perspective, knowledge is one of the regulative structures of human behavior, along with other mental and societal structures such as institutions, all of them fashioned by given material conditions. At the same time, human actions externalize these structures in transformations of their material environment. These changes may then be internalized again in human behavioral and societal structures, giving rise to an interplay of "externalization" and "internalization."

\footnotetext{
${ }^{21}$ For the concept of niche construction, see John Odling-Smee, Douglas H. Erwin, Eric P. Palkovacs, Marcus W. Feldman, and Kevin N. Laland, "Niche Construction Theory: A Practical Guide for Ecologists," The Quarterly Review of Biology 88, no 1 (2013): 4-28.

22 See Manfred D. Laubichler and Jürgen Renn, "Extended Evolution: A Conceptual Framework for Integrating Regulatory Networks and Niche Construction," Journal of Experimental Zoology Part B: Molecular and Developmental Evolution 324, no 7 (2015): 565-577; Jürgen Renn, "From the History of Science to the History of Knowledge - and Back," Centaurus 57 (2015): 37-53.
}

\section{HoST - Journal of History of Science and Technology 12, pp. 1-22 \\ DOI 10.2478/host-2018-0001}


This interplay shows remarkable characteristics: First, among any historically given conditions, societies are capable of reproducing some of them and others not. For instance, in the course of the Neolithic Revolution, humans learned to reproduce environmental conditions allowing them to produce their own food. In this way, accidental external conditions, such as the local availability of plants and animals capable of being domesticated, could become indispensable features of further global development, accounting for the path dependence of the process. ${ }^{23}$

Second, a given physical environment and material culture open up specific possibilities for the development of regulative structures. The "horizon of possibilities" shaped by these material conditions may leave room for their exploration. ${ }^{24}$ In particular, as the material conditions and external manifestations of human social and behavioral structures play a key role in their regulation, such an exploration will affect these societal structures in turn.

Exploring the horizon offered by the material means and external representations of a knowledge system may lead to a transformation of the system. Exploring the possibilities of social organization offered by a particular material culture may similarly engender societal transformations. Needless to say, neither kind of transformation is ever simple or straightforward, but both are worthy of deeper investigation from a common perspective. Like biological evolution, cultural evolution is an irreversible, singular, and unrepeatable process whose outcomes cannot be predicted.

Historically, the accessibility of fossil energy resources was an accidental, local condition fostering the emergence of industrialized societies. In the long run, however, it will not be possible to maintain the conditions it offered for societal development, let alone to

\footnotetext{
${ }^{23}$ For a discussion of the role of symbolic constructions in the context of the Neolithic Revolution, see Trevor Watkins, "New Light on Neolithic Revolution in South-west Asia," Antiquity 84 no 325 (2010): 621-634.

${ }^{24}$ See Edmund Husserl, Erfahrung und Urteil: Untersuchungen zur Genealogie der Logik, 27 (Prague: Academia Verlagsbuchhandlung, 1939). The term in the sense used here was introduced in Peter Damerow and Wolfgang Lefevvre, "Arbeitsmittel der Wissenschaft: Nachbemerkung zur Theorie der Wissenschaftsentwicklung," in Rechenstein, Experiment, Sprache: historische Fallstudien zur Entstehung der exakten Wissenschaften, ed. Peter Damerow and Wolfgang Lefevre, 223-233 (Stuttgart: Klett-Cotta, 1981).
} 
reproduce them. The seemingly cheap and limitless availability of fossil resources has, for a time, decoupled industrialized societies from local environmental conditions. The abundance of energy has decontextualized their regulative structures, thus suggesting that they have a universal character, that is, a character can be superimposed on arbitrary local conditions and projected into an indefinite future. Now the time for such a "local universalism" is over. ${ }^{25}$ The consequences of industrialization restore a regulatory function to the environment on a global scale that will affect human behavioral and societal structures, albeit in locally diverse ways.

The inevitable transformation of fossil fuel based societies will have to be an exploratory process as well. Because locally variables will again become increasingly relevant, there will be no one-size-fits-all solution. Future solutions to energy problems will probably no longer be able to deal with technical systems independently from local and global environmental and societal contexts. A new knowledge economy will have to be based on the perspective of "global contextualism," ${ }^{26}$ paying attention to both locally varying contexts and global consequences of any human intervention in the Earth system. Therefore, a new knowledge economy will have to deal with the "borderline problems" at the interfaces between the human ergosphere and other spheres of the Earth system. And it will have to provide for modes of integrating the legacy of global human knowledge with the new local knowledge emerging at these interfaces.

\section{Concluding Remarks}

We need, in particular, more knowledge about equilibria in global dynamic systems, but also cultural abstractions that help us to maintain or to adapt these equilibria by representing them locally in the lives and minds of human actors. In cultural evolution, the internalization and reproduction of given external conditions within societal

${ }^{25}$ On local universalism, see Yehuda Elkana, "The University of the 21st Century: An Aspect of Globalization," in The Globalization of Knowledge in History, ed. J. Renn, 605-630, 610 (Berlin, Germany: Edition Open Access, 2012): http://edition-open-access.de/studies/1/29/index.html (accessed September, 5, 2018).

${ }^{26}$ Elkana, "The University of the 21st Century," 611-612.

HoST - Journal of History of Science and Technology 12, pp. 1-22

DOI 10.2478/host-2018-0001 
development was largely a matter of circumstance. In epistemic evolution, it will have to become more and more a matter of knowledge. The sense of urgency driven by the awareness that we are living in the Anthropocene should thus concern not only politics and economies, but also the quest for more knowledge that may trigger crossscale effects on social behavior.

HoST - Journal of History of Science and Technology 12, pp. 1-22

DOI 10.2478/host-2018-0001 